\title{
Neural mechanisms underlying neurooptometric rehabilitation following traumatic brain injury
}

\author{
This article was published in the following Dove Press journal: \\ Eye and Brain \\ 18 January 2012 \\ Number of times this article has been viewed
}

\author{
Caitlin M Hudac' \\ Srinivas Kota' \\ James L Nedrow ${ }^{2}$ \\ Dennis L Molfese ${ }^{1,3}$ \\ 'Department of Psychology, University \\ of Nebraska-Lincoln, ${ }^{2}$ Oculi Vision \\ Rehabilitation, ${ }^{3}$ Center for Brain, \\ Biology, and Behavior, University \\ of Nebraska-Lincoln, Lincoln, NE
}

Correspondence: Dennis L Molfese Department of Psychology, University of Nebraska-Lincoln, 238 Burnett Hall, Lincoln, NE 68588

Tel +l 4024727976

Fax + I 4024724637

Email dlmolfese@mac.com
Abstract: Mild to severe traumatic brain injuries have lasting effects on everyday functioning. Issues relating to sensory problems are often overlooked or not addressed until well after the onset of the injury. In particular, vision problems related to ambient vision and the magnocellular pathway often result in posttrauma vision syndrome or visual midline shift syndrome. Symptoms from these syndromes are not restricted to the visual domain. Patients commonly experience proprioceptive, kinesthetic, vestibular, cognitive, and language problems. Neurooptometric rehabilitation often entails the use of corrective lenses, prisms, and binasal occlusion to accommodate the unstable magnocellular system. However, little is known regarding the neural mechanisms engaged during neurooptometric rehabilitation, nor how these mechanisms impact other domains. Event-related potentials from noninvasive electrophysiological recordings can be used to assess rehabilitation progress in patients. In this case report, high-density visual event-related potentials were recorded from one patient with posttrauma vision syndrome and secondary visual midline shift syndrome during a pattern reversal task, both with and without prisms. Results indicate that two factors occurring during the end portion of the P148 component (168-256 milliseconds poststimulus onset) map onto two separate neural systems that were engaged with and without neurooptometric rehabilitation. Without prisms, neural sources within somatosensory, language, and executive brain regions engage inefficient magnocellular system processing. However, when corrective prisms were worn, primary visual areas were appropriately engaged. The impact of using early neurooptometric rehabilitation for posttrauma vision syndrome, visual midline shift syndrome, and other similar subtle vision disorders to support neural reorganization is discussed.

Keywords: traumatic brain injury, posttrauma vision syndrome, visual midline shift syndrome, visual event-related potentials, source localization, neural reorganization

\section{Introduction}

Each year an average of 1.4 million individuals in the United States suffer a traumatic brain injury (TBI). TBI is often caused by a physical blow to the head or whiplash that results in an abrupt, trauma-induced physiological disruption of brain function. ${ }^{1}$ Changes in rotational forces and acceleration-deceleration can damage brain tissue at both the neuronal and axonal levels. ${ }^{2,3}$ Recent innovations using neuroimaging techniques such as diffusion tensor imaging can identify injury to white matter tracts. ${ }^{4}$ However, even in moderate to severe cases of TBI, neuroradiological examinations can appear normal despite persistent behavioral symptoms. Frequently, subtle sensory problems are often overlooked as contributors to poor performance..$^{5-7}$

Vision problems are among the most common and pervasive TBI symptoms. ${ }^{8}$ Two examples of visual dysfunction related to TBI are posttrauma vision syndrome (PTVS) 
and visual midline shift syndrome (VMSS), ${ }^{9,10}$ PTVS is characterized by exotropia, exophoria, convergence and accommodative insufficiency, oculomotor dysfunction, and increased myopia. ${ }^{10}$ Patients often report symptoms such as diplopia, blurred near vision, eye strain (asthenopia), sensitivity to light (photophobia), and perceived movement of print or stationary objects. Related more to balance problems, VMSS is characterized by hemiplegia, hemiparesis, flexion, extension, and side neglect. These patients report walls appearing to move and tilted floors. The patient's center of gravity will be shifted, often resulting in the patient leaning away from the affected side.

Both syndromes occur frequently following TBI and reflect widespread dysfunction across vision and other sensory domains. One possible explanation is that the visual system heavily influences other systems. There certainly is ample evidence of a connection between the visual and vestibular systems. ${ }^{11,12}$ One primary function of the vestibular system is to provide compensatory eye movement to correspond with head motion (eg, ocular reflex). The two systems are yoked together for stabilization of the visual scene while the head is in motion. Considering proprioceptive and kinetic systems, Padula and colleagues ${ }^{13}$ suggested that vision is the primary determinate of the visual egocenter (eg, visual midline), noting that yoked prisms successfully corrected posture and balance in patients with VMSS.

Impairment to the magnocellular visual pathway may lead to the symptoms evident in PTVS, VMSS, and other TBI-related visual dysfunctions. The magnocellular ambient pathway establishes a visual midline that matches other sensory inputs. Without the proper signals, interference can occur between the incorrect concept of the visual midline and sensory-motor functions, resulting in the multimodal symptoms of PTVS and VMSS (eg, spatial disorientation, impaired balance and posture, and poor visual memory and attention). Dysfunction within the ambient system is often best diagnosed using visually evoked potentials. This technique uses three electrodes placed on the scalp above the occipital and posterior frontal midline to detect changes in the P100 component while patients observe a checkerboard reversal task with and without corrective prisms and lenses. In PTVS, when prisms are introduced, the amplitude of the P100 component of visually evoked potentials increases. One interpretation is that the prisms are enhancing organization and promoting feed-forward spatial information to the primary occipital visual regions. ${ }^{9}$

Despite the ability to use electrophysiological recordings for the assessment of cognitive impairments and evaluate prognosis, ${ }^{14-16}$ little is known about the mechanisms of rehabilitation. Evidence from severely impaired TBI patients during a pattern reversal task suggests that the distributed visual networks desynchronize across brain regions. ${ }^{17}$ While corrective prisms, lenses, and sectoral or binasal occlusion often lead to marked functional improvements in TBI and stroke patients, ${ }^{18,19}$ the neural mechanisms engaged in such changes remain unknown.

The purpose of the present case study was to better understand how neurooptometric rehabilitation following TBI improves neural functioning. Visual event-related potentials (VERPs) were recorded from a patient with PTVS and secondary VMSS. She completed a pattern reversal VERP task under two different conditions: (1) with normal lenses (no prisms condition), and (2) with corrective prisms. A recent study utilizing electroencephalography and functional magnetic resonance imaging indicated that the early N75/P85 component elicited by the pattern reversal task was generated from primary visual cortex. ${ }^{20}$ Although studies have investigated behavioral changes with use of prisms in patients following a cerebrovascular accident, ${ }^{13}$ to date no studies utilized neuroimaging to determine if prisms engage the same neural mechanisms during the pattern reversal task. The patient contacted the research team after learning about ongoing work involving TBI. The University of Lincoln-Nebraska's Institutional Review Board approved this study, and the patient provided informed consent before participating.

\section{Case presentation}

A female, aged 44 years, was referred to a local neurooptometrist following a head injury 11 months before VERP testing. Prior to TBI, the patient exhibited no signs of neurological impairment or major health concerns, aside from a shoulder and clavicle injury. Her head injury occurred when the patient fell in the shower, striking her head on a toilet, bathtub, and floor tiles in rapid succession. An undetermined period of unconsciousness and paralysis immediately followed, after which the patient was able to stand and walk with some hesitancy that improved over a brief time. She did not seek immediate medical attention, but after experiencing increasingly severe headaches over several hours sought out an urgent care facility that subsequently diagnosed her with a closed head injury. Continued symptoms, including headache, dizziness, short-term memory loss, frequent falls due to poor balance and depth perception, and nausea, left her unable to return to work. Magnetic resonance imaging and computed tomography scans were reported as unremarkable. Headaches described as throbbing and stinging ranged from 
moderate (6/10) to severe (9.9/10) on the Migraine Disability Assessment. ${ }^{21}$ She localized this pain to the left frontal, bilateral occipital, and occasionally bilateral temporal regions. She reported diminished sleep, averaging approximately 4 hours a night. Visual problems consisted of phonophobia, blurred vision, sensitivity to bright light (photophobia), visual memory problems, and occasional episodes of diplopia. She also reported extreme difficulty with reading and reported seeing words "run off the page." Aggressive physical therapy, occupational therapy, and speech therapy were sought. However, many of the symptoms persisted.

Prior to the injury, the patient was active in her career and known for abilities as an expert ambidextrous marksman. Upon retrospective completion of the Edinburgh Handedness Inventory ${ }^{22}$ and subsequent verification by her husband, before the injury she scored as ambidextrous with slight left-handed tendencies (mean laterality quotient $=-0.25$, where +1.00 indicates that all tasks are performed exclusively with the right hand and -1.00 indicates that all tasks are performed only with the left hand). On the day of VERP testing, the Edinburgh Handedness Inventory indicated that she was primarily lefthanded due to her inability to execute fine motor movements using her right hand (mean laterality quotient $=-0.75$ ).

Her neurooptometrist confirmed a diagnosis of PTVS and secondary VMSS. These results were verified by visually evoked potentials test and Clinical Test of Sensory Interaction and Balance (Table 1). In addition, examination indicated a two-prism diopter left hypertropia secondary to partial paresis of the third cranial nerve. Hyperacusis was also diagnosed. Therapeutic prisms, both eyes (1 diopter in), including midpupil binasal occlusion in a clipon frame with additional corrective refractive lenses were prescribed right eye (3/4 diopter up), left eye (3/4 diopter down) for diplopia. After a subsequent fall, prism strength was changed to 3 diopters up and in ( 45 degrees) right eye and 3 diopters up and out (45 degrees) left eye with continued binasal occlusion (clipon frames). This prescription was used during VERP testing.

\section{Visual acuity}

Five months prior to VERP session, the patient's best corrected visual acuity initially tested as 20/100 (left eye), 20/40 (right eye), and 20/70 (both eyes). Corrected vision with lenses and prisms was 20/20 (left eye), 20/20 (right eye), and 20/15 (both eyes).

\section{Overall visual perceptual ability}

The patient completed the Motor-Free Visual Perception Test, Third Edition ${ }^{23}$ twice on the same day 3 months prior
Table I Positive diagnostic results for posttrauma vision syndrome and visual midline shift syndrome

\begin{tabular}{|c|c|c|}
\hline & No prisms & With prisms \\
\hline \multicolumn{3}{|l|}{ Visual evoked response } \\
\hline \multicolumn{3}{|l|}{ Low-density checkerboard } \\
\hline Amplitude & $4.92 \mu \mathrm{V}$ & $\mathrm{I} 3.2 \mu \mathrm{V}$ \\
\hline Latency & 40 milliseconds & I6.66 milliseconds \\
\hline \multicolumn{3}{|l|}{ High-density checkerboard } \\
\hline Amplitude & $4.38 \mu \mathrm{V}$ & $9.95 \mu \mathrm{V}$ \\
\hline Latency & 88.33 milliseconds & 21.66 milliseconds \\
\hline \multicolumn{3}{|l|}{ Modified CTSIB } \\
\hline COG alignment & Right, $34 \%$ & Front, $20 \%$ \\
\hline \multicolumn{3}{|l|}{ Mean COG sway velocity } \\
\hline Firm stand-eyes open & 2 degrees/second & 0.4 degrees/second \\
\hline Firm stand-eyes closed & 2.5 degrees/second & 0.6 degrees/second \\
\hline Foam stand-eyes open & 2.7 degrees/second & I.5 degrees/second \\
\hline Foam stand-eyes closed & - & 2.4 degrees/second \\
\hline
\end{tabular}

Notes: Patient was unable to complete the foam stand-eyes closed in no prisms condition. During the visual evoked response diagnostic test, both low-and highdensity checkerboards were presented while visual event-related potentials were recorded. With prisms, amplitudes were approximately $8 \mu \mathrm{V}$ higher and occurred 25 milliseconds faster than the no prisms condition. These results are consistent with positive diagnosis for posttrauma vision syndrome. During the modified Clinical Test of Sensory Interaction and Balance, the patient stood on either a firm or foam platform and was asked to maintain her balance with her eyes open or closed. Results indicate substantially less sway velocity with prisms compared to the no prisms condition. Altogether, center of gravity alignment decreased from $34 \%$ in the no prisms condition to $20 \%$ in the with prisms condition. These results are consistent with positive diagnosis for visual midline shift syndrome.

Abbreviations: COG, center of gravity; CTSIB, Clinical Test of Sensory Interaction of Balance.

to VERP testing. First, without corrective prisms, the patient scored a standard score of $<55$ (raw score $=33,<1 \%$ rank). The patient then completed the task with corrective prisms and scored a standard score of 137 (raw score $=63,99 \%$ rank). These results corresponded to previous tests suggesting that her vision functions and visual-perceptual abilities were positively correlated. ${ }^{24}$

\section{Speech and language}

Without prisms, the patient's speech was highly affected. A pronounced stutter occurred during virtually every vocalization, with five to eight rapid repetitions of initial syllables before final word completion. However, the patient appeared largely unaware of the stutter. Language comprehension was not affected, although the patient noted her own struggle to recall the correct words. Within 3 minutes of putting the prisms back on, her speech fluency improved markedly although the stutter did not disappear entirely ( $\sim 80 \%$ clear speech).

\section{Balance and walking}

Without the prisms, the patient was assisted to the VERP testing room in a wheelchair due to her inability to walk without periodically falling. At the end of the test session, wearing the 
A

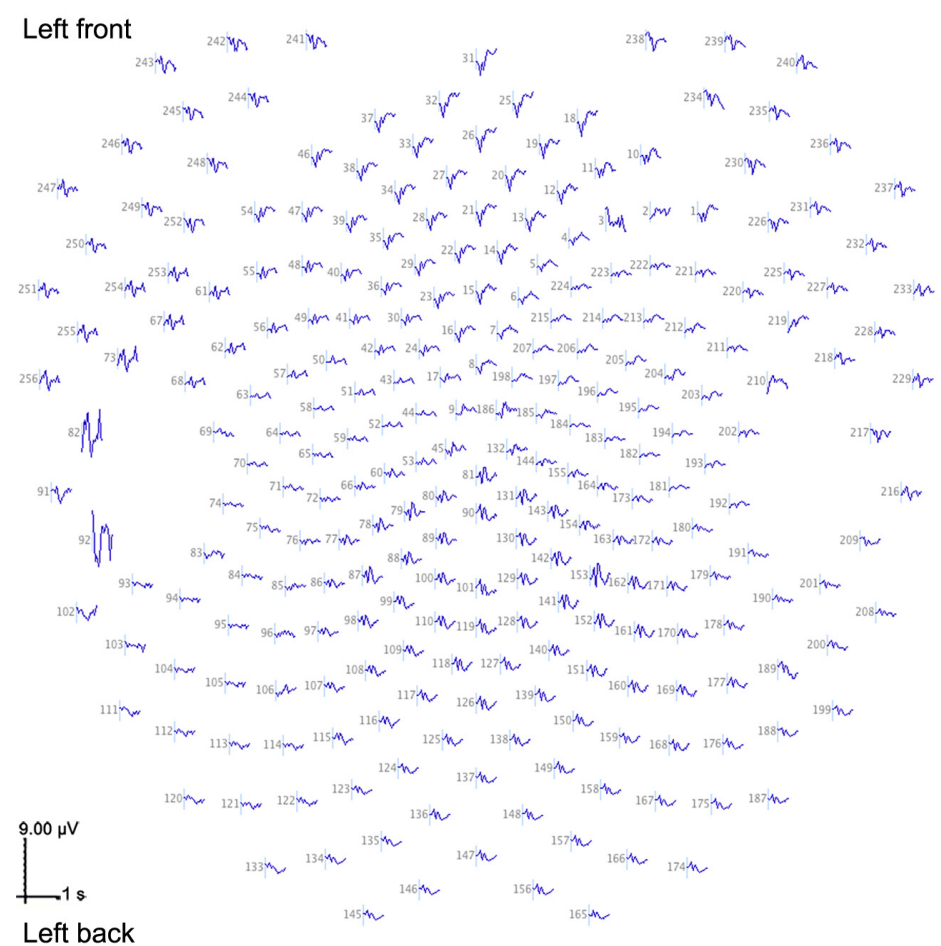

B

With prisms

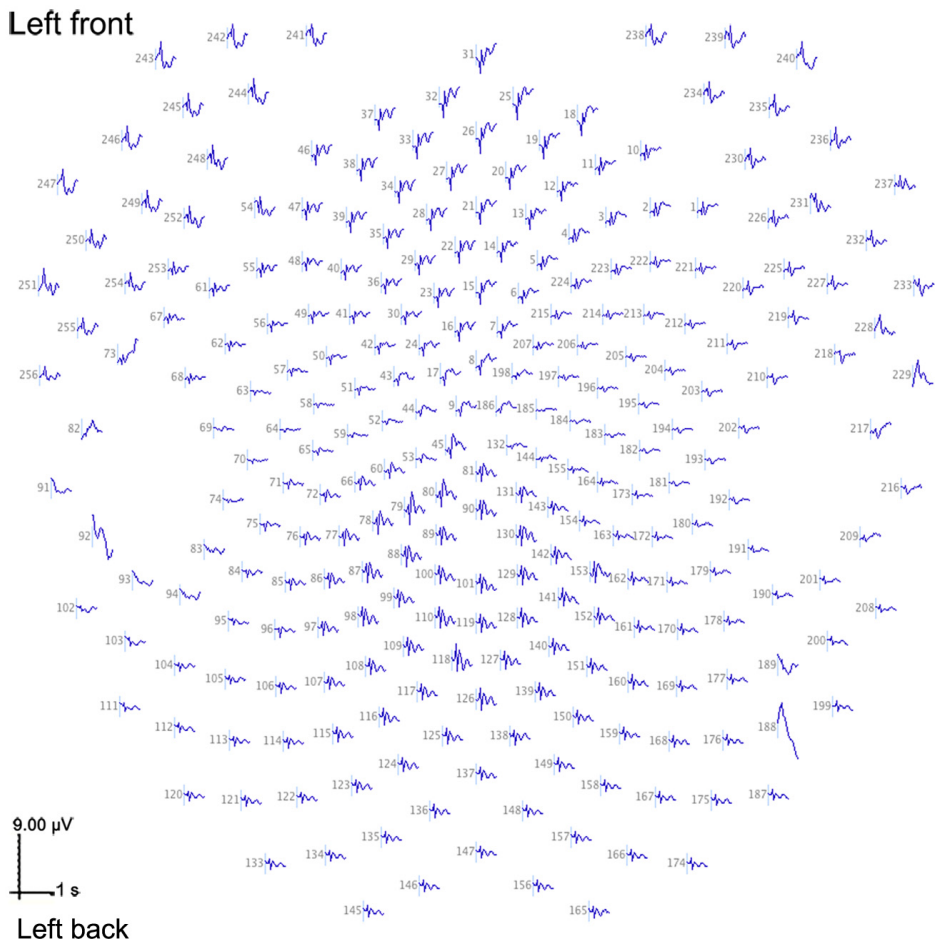

Figure I Epoch-averaged visual event-related potential recordings from both conditions for 256-electrode high-density array. The front of the head is at the top of each sphere with the left hemisphere on the left. Positive voltage is displayed up. Time course is 800 milliseconds from stimuli onset. The amplitudes of visual event-related potentials for the no prisms condition (A) are much smaller than for the with prisms condition (B). In addition, the visual event-related potentials appear much better organized for the with prisms condition across the scalp, particularly in frontal and centroparietal regions. 
prisms, she stood up from the chair without help and walked down a narrow hall with a normal gait to use a hairdryer.

\section{Behavior}

Researchers observed drastic differences in mood with and without the prisms. Without prisms, her demeanor appeared to be anxious, she moved restlessly in her chair, and her eyes rapidly darted from side-to-side as she observed items in front of her. During the electrode net application, she sat silent and answered direct questions with minimal vocalizations. When she wore the prisms, her behavior changed abruptly. She no longer appeared anxious, her gaze was markedly steady, and she initiated friendly conversation and laughed. Altogether, she appeared more relaxed throughout the remainder of the session.

\section{Method}

Testing was conducted using a 256 silver/silver chloride highdensity electrode array to record VERPs using Net Station ${ }^{\text {R }}$ 4.4.2 software (Electric Geodesics Inc, Eugene, OR). In a within-subject design, the patient completed the first run of the VERP checkerboard pattern reversal task with corrective lenses that did not contain the prisms. For complete details of stimuli and procedures, see the Supplementary Data section. Next, prisms were attached as a clipon to the patient's lenses while she remained in the electrode net during a 30-minute break between tests. The purpose of this break was to allow ample time for the patient to visually adjust to the new prisms. Once this interval ended, a second presentation of the VERP task occurred.

\section{Results}

After preprocessing the raw signal (see Supplemental Data section), 69 single-trial epochs were included in the analysis. VERP trial data averaged across all epochs are displayed in Figure 1 for both the no prisms and with prisms conditions. Next, a series of three distinct analysis approaches were used to analyze the VERP data obtained from the patient: (1) a principal components analysis analysis of variance (ANOVA) procedure, (2) a dynamic channel selection strategy, and (3) a neural source analysis. These three approaches and their results are described in order below.

\section{Principal components analysis - ANOVA}

In this procedure, the principal components analysis serves as a multivariate, independent method to extract temporal components or factors that accounted for the maximum variability in the single-trial epochs. The output of this analysis serves as the dependent measures in an ANOVA procedure that assessed the statistical significance of these factors between conditions. In this way, temporal factors associated with particular time windows of the VERP were derived that significantly differed between conditions (eg, no prisms versus with prisms). ${ }^{25}$

All single-trial epochs were submitted to the temporal principal components analysis in which the 200 time points (sampled at 4 milliseconds intervals) were treated as variables and individual VERP trials were treated as cases. Factors were orthogonally rotated using varimax rotation. After applying an eigenvalue greater than 1.0 criterion, ${ }^{26}$ nine factors that accounted for $92.12 \%$ of the total variability in the data set were identified for subsequent analyses. Bartlett component scores from the principal components analysis were submitted to an ANOVA to identify the sources of variability in the VERPs. The ANOVA design included repeated measures for condition (two: no prisms, with prisms) and

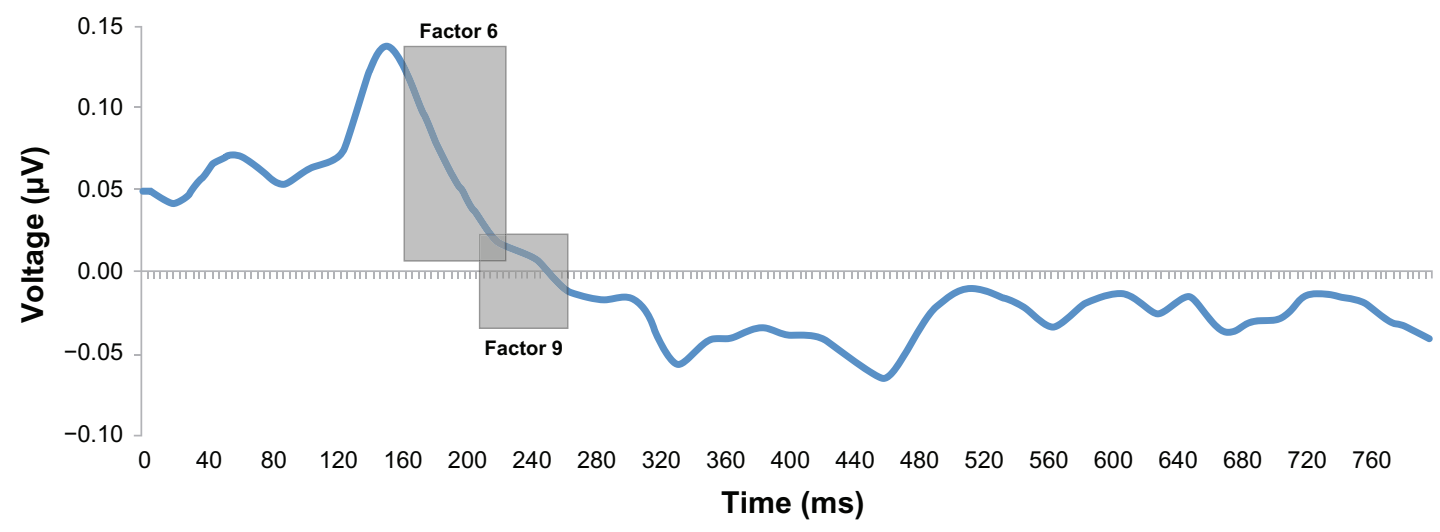

Figure 2 Grand average of visual event-related potentials of all trials for both conditions. Temporal factor 6 and factor 9 are displayed in gray boxes from $168-256$ milliseconds, highlighting the end portion of the PI48 component. Calibration marker is $-0.1 \mu \mathrm{V}$ to $0.15 \mu \mathrm{V}$ with positive voltage up. Time course is 800 milliseconds from stimuli onset.

Abbreviation: ms, milliseconds. 


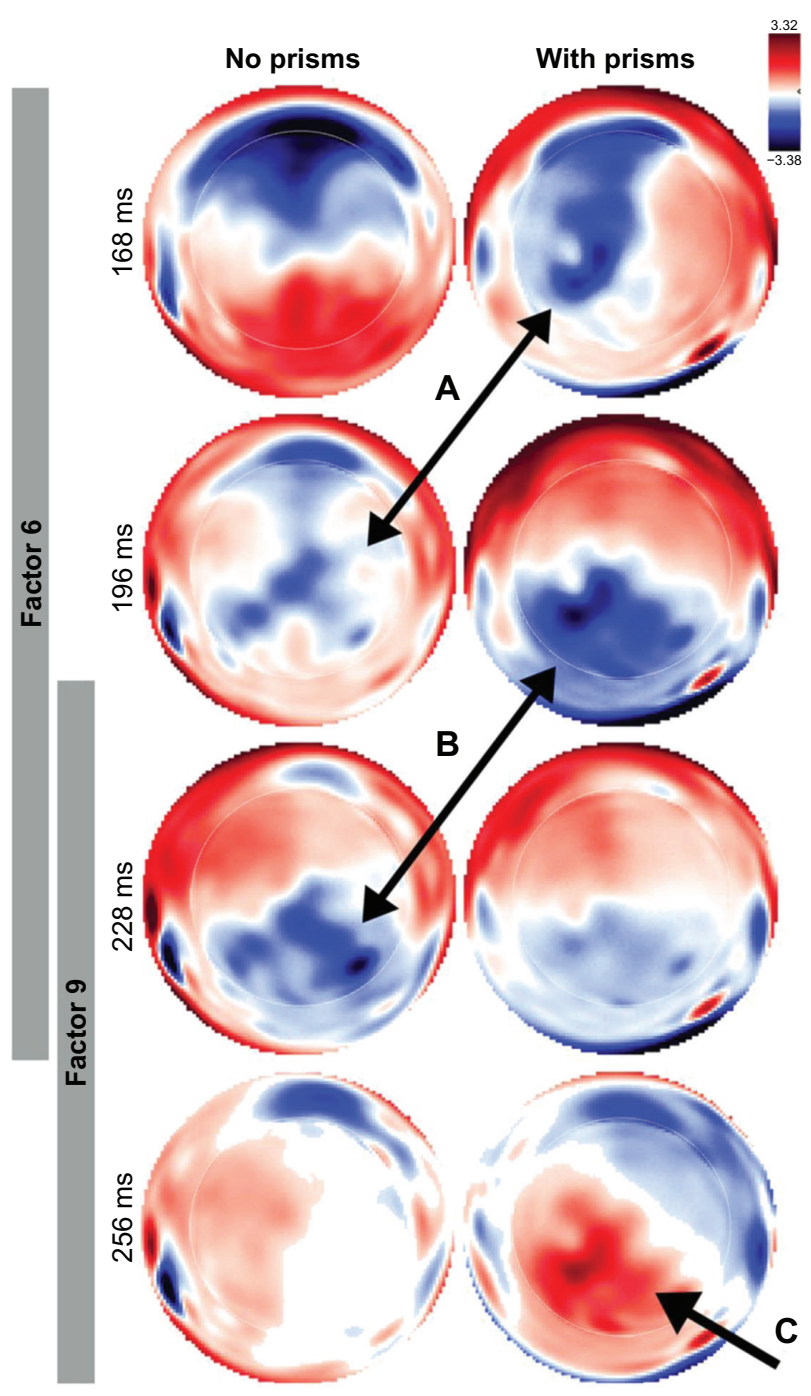

Figure 3 Scalp topographies for temporal factor 6 (168-228 milliseconds from stimuli onset) and factor 9 (208-228 milliseconds from stimuli onset). Anterior scalp locations appear at the top of the sphere. Posterior scalp locations appear at the bottom of the sphere. Color map ranges from peak positivity (red; $3.32 \mu \mathrm{V}$ ) to peak negativity (blue; $-3.35 \mu \mathrm{V}$ ). Bidirectional arrows (A) and (B) highlight peak positivity that appear in the with prisms condition 30-45 milliseconds before the no prisms condition. Arrow (C) indicates a peak positivity appearing at the end of the time frame in with prisms condition, but not the no prisms condition.

Abbreviation: ms, milliseconds.

electrode scalp regions (18: left orbital, right orbital, left inferior frontal, right inferior frontal, left prefrontal, right prefrontal, left inferior temporal, right inferior temporal, left temporal, right temporal, left temporoparietal, right temporoparietal, left parietal, right parietal, left inferior occipital, right inferior occipital, left occipital, right occipital) using Greenhouse-Geisser correction.

The results reported below focused only on the principal components analysis components that differed between the no prisms and with prisms conditions. ANOVA identified a main effect of condition for factor $9[\mathrm{~F}(1,68)=5.67$, $P=0.02$, observed power $=0.651, \mathrm{~d}=0.29]$ and an electrode region $\mathrm{x}$ condition interaction for factor $6[\mathrm{~F}(17$, $1156)=2.67, P=0.04$, observed power $=0.694, \mathrm{~d}=0.29]$. Figure 2 illustrates these temporal factors in gray boxes superimposed on the centroid or grand average waveform that was averaged across all 138 trials. Together, factor 6 (168-228 milliseconds) and factor 9 (208-256 milliseconds) characterize VERP activity that occurred near the late portion of the P148 component (168-256 milliseconds). The scalp topographies for each of these factors are illustrated in Figure 3 at intervals corresponding to the beginning, peak, and end latencies for each factor indicated in the gray boxes. Peaks of negativity (illustrated in blue) shift from anterior (front of the head is at the top of sphere in the figure) to posterior scalp locations (back of the head). The bidirectional arrows, (a) and (b), indicate latency differences between conditions, in which negative peaks reliably appeared 30-45 milliseconds earlier in the with prisms condition than in no prisms condition. At the end of the time frame, arrow (c) indicates a positive voltage shift (illustrated in red) that occurred in the with prisms condition but not during the no prisms condition.

\section{Dynamic channel selection}

To further investigate the specificity of the VERP difference between the lens-no lens conditions, a dynamic channel selection strategy was implemented which employed spatiotemporal modeling, dynamic channel selection, univariate classification, and decision fusion. ${ }^{27}$ The goal of dynamic channel selection strategy was to identify spatiotemporal elements that carried the most discriminatory information for correctly classifying different brain responses. For each observation, 69 single-trial spatiotemporal VERP arrays were considered. The average spatiotemporal arrays in Figure 4 illustrate which of the electrode sites had peak distribution differences for each condition across time. From 51,200 possible electrode site $\mathrm{x}$ time spatiotemporal elements, 1285 elements best represented the differences between conditions and were submitted to the classification procedure. Half of the data were used for training and the remaining half to test classification accuracy. Each sample was classified using a Gaussian classifier and decisions were fused into a single fusion vector. Results indicate that VERPs recorded during the no prisms condition were accurately classified $87.56 \%$ while the with prisms condition was accurately classified $87.09 \%$. Overall, average classification accuracy was $87.32 \%$. Such high levels of distinct classifications indicate that the VERPs reliably differed between the two conditions. 
A

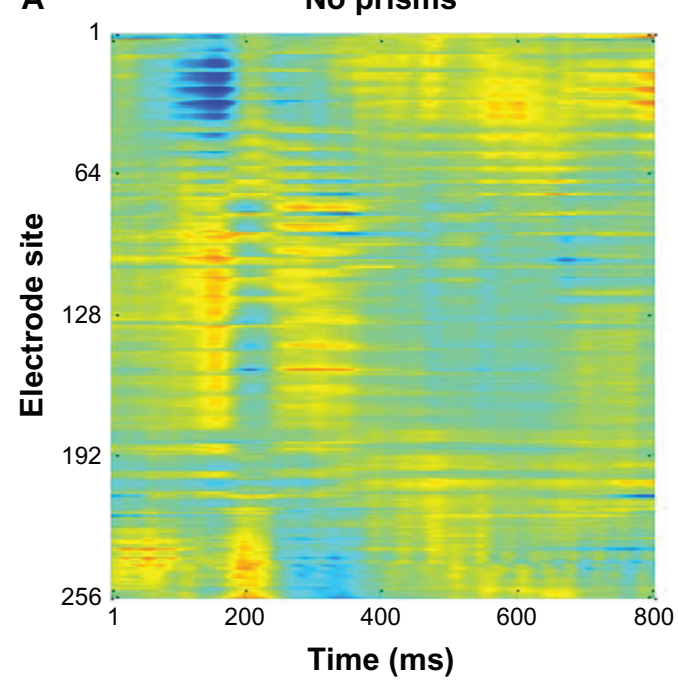

B

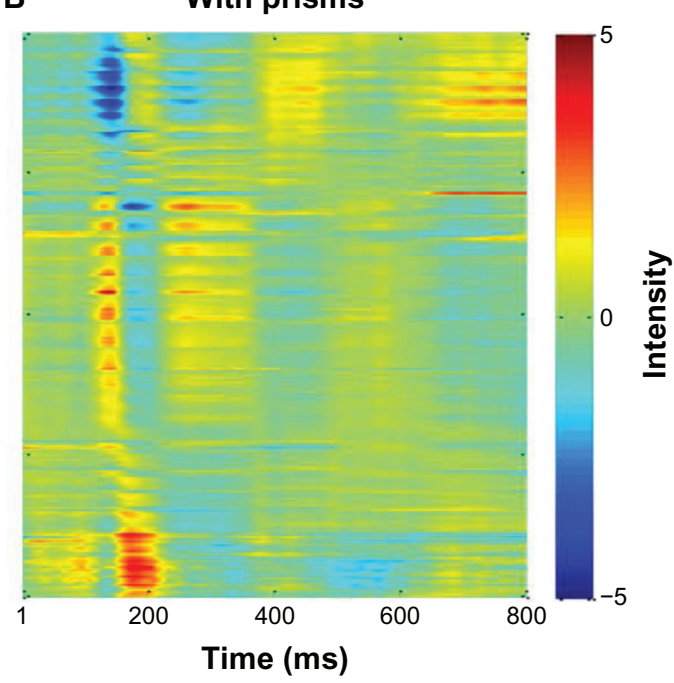

Figure 4 Averaged spatiotemporal arrays for no prisms $(\mathbf{A})$ and with prisms $(\mathbf{B})$ conditions. Each coordinate on the grid represents the intensity (range $=-5$ to 5 ) of the average spatiotemporal element for each electrode site at each time point from stimulus onset. A drastic difference in intensity between conditions (with prisms $>$ no prisms) is evident following the positive peak of the PI48 component ( 150-215 milliseconds), particularly in electrode sites 230-256.

Abbreviation: ms, milliseconds.

\section{Source analysis}

As a final analysis step, brain source analyses were conducted to estimate the neural sources responsible for generating the scalp recorded VERPs that varied between conditions. With high-density VERPs it is possible to produce source analysis maps of cortical activation superimposed on an adult magnetic resonance imaging template brain. Single trials were input to separate analyses for each condition into the GeoSource ${ }^{\circledR}$ software program (v2.0; Electrical Geodesics) employing the standardized low resolution brain electromagnetic tomography solution (see Supplemental Data for details). From the timeframes identified by the temporal principal components analysis, source waveforms within each Brodmann's area were generated using a finite difference model. These source waveforms were then analyzed using mean amplitude measures (nA) within each Brodmann's area, averaged over the timeframe of each temporal factor. A one-way ANOVA analyzed whether the estimated source activations differed significantly between conditions.

The source activation maps for each condition were compared for each voxel to determine if differential neural networks were engaged and generated the VERPs. Source estimation results for factor 6 (158-228 milliseconds) identified brain sources distributed primarily in the anterior superior temporal gyrus for both conditions. However, despite similar regional activation, sources were statistically less active during the no prisms condition (peak intensity $=1.60 \mathrm{nA}$ ) than the with prisms condition (peak intensity $=3.29 \mathrm{nA}$ ).
Factor 9 (208-256 milliseconds) identified brain sources that were distributed in the parahippocampal gyrus and inferior temporal gyrus for both conditions. Additional activation for the with prisms condition was identified in the limbic lobe. Although both conditions show maximum activation in the left hemisphere (no prisms peak $=1.75 \mathrm{nA}$; with prisms peak $=1.76 \mathrm{nA}$ ), the pattern of activation only occurred bilaterally in both hemispheres during the with prisms condition.

A one-way ANOVA identified significant differences between conditions for certain Brodmann's area sources. These results are reported for the no prisms and with prisms conditions in Tables 2 and 3, respectively. Activation during the no prisms condition was more distributed across the brain than the with prisms condition. In other words, more brain volumes were activated during the no prisms condition. ${ }^{28}$ Altogether, 29 Brodmann's areas had a greater magnitude of activation during the no prisms condition. Importantly, these activated areas included somatosensory, language, and executive regions, which are not typically activated during primary encoding of visual information. Primary visual areas (eg, V1, V2, and V3) are more engaged during the with prisms condition relative to the no prisms condition, suggesting that the prism intervention optimized the visual processing system. Figure 5 highlights the different activation patterns of the conditions superimposed on the Montreal Neurological Institute template brain. Specifically, a widely distributed network across frontal, temporal, and parietal regions were engaged during the no prisms condition, whereas a more focused neural network centered 
Table 2 Activation areas identified using GeoSource ${ }^{\circledR}$ software program (v2.0; Electrical Geodesics Inc, Eugene, OR) for no prisms condition

\begin{tabular}{|c|c|c|c|c|c|c|}
\hline \multirow[t]{2}{*}{ Region } & \multirow[t]{2}{*}{ BA } & \multirow[t]{2}{*}{ Hemi } & \multicolumn{2}{|c|}{ Factor 6} & \multicolumn{2}{|l|}{ Factor 9} \\
\hline & & & F value & $P$ & F value & $P$ \\
\hline \multicolumn{7}{|l|}{ Vision and somatosensory } \\
\hline \multirow[t]{4}{*}{ Primary somatosensory cortex } & 1 & $\mathrm{~L}$ & 5.157 & 0.025 & 5.295 & 0.023 \\
\hline & 1 & $\mathrm{R}$ & 34.770 & 0.000 & 34.470 & 0.000 \\
\hline & 2 & $\mathrm{~L}$ & 5.392 & 0.022 & 5.528 & 0.020 \\
\hline & 3 & $\mathrm{~L}$ & 20.832 & 0.000 & 18.409 & 0.000 \\
\hline \multirow[t]{3}{*}{ Primary motor cortex } & 3 & $\mathrm{R}$ & 8.310 & 0.005 & 7.011 & 0.009 \\
\hline & 4 & $\mathrm{~L}$ & 15.693 & 0.000 & 13.610 & 0.000 \\
\hline & 4 & $\mathrm{R}$ & 8.303 & 0.005 & 7.673 & 0.006 \\
\hline \multirow[t]{2}{*}{ Premotor cortex } & 6 & $\mathrm{~L}$ & 15.287 & 0.000 & 10.637 & 0.001 \\
\hline & 6 & $\mathrm{R}$ & 9.543 & 0.002 & 7.546 & 0.007 \\
\hline \multirow[t]{3}{*}{ Frontal eye fields } & 8 & $\mathrm{~L}$ & 13.779 & 0.000 & 11.741 & 0.001 \\
\hline & 8 & $\mathrm{R}$ & 35.699 & 0.000 & 35.108 & 0.000 \\
\hline & 42 & $\mathrm{~L}$ & 8.202 & 0.005 & 9.495 & 0.002 \\
\hline \multirow[t]{3}{*}{ Primary gustatory cortex } & 42 & $\mathrm{R}$ & 18.513 & 0.000 & 18.017 & 0.000 \\
\hline & 43 & $\mathrm{~L}$ & 11.987 & 0.001 & 12.829 & 0.000 \\
\hline & 43 & $\mathrm{R}$ & 26.658 & 0.000 & 27.368 & 0.000 \\
\hline \multicolumn{7}{|l|}{ Language } \\
\hline Angular gyrus (Wernicke's area) & 39 & $\mathrm{~L}$ & 5.773 & 0.018 & 5.948 & 0.016 \\
\hline Superior temporal gyrus (Wernicke's area) & 22 & $\mathrm{~L}$ & - & - & 4.520 & 0.035 \\
\hline \multicolumn{7}{|l|}{ Pars opercularis (Broca's area) } \\
\hline & 44 & $\mathrm{~L}$ & 4.515 & 0.035 & 5.604 & 0.019 \\
\hline \multirow[t]{2}{*}{ Pars triangularis (Broca's area) } & 44 & $\mathrm{R}$ & 34.883 & 0.000 & 33.315 & 0.000 \\
\hline & 45 & $\mathrm{R}$ & 41.465 & 0.000 & 40.343 & 0.000 \\
\hline \multicolumn{7}{|l|}{ Executive and memory } \\
\hline Anterior prefrontal cortex & 10 & $\mathrm{R}$ & 24.390 & 0.000 & 23.079 & 0.000 \\
\hline Middle temporal gyrus & 21 & $\mathrm{R}$ & 24.876 & 0.000 & 22.943 & 0.000 \\
\hline \multirow[t]{4}{*}{ Dorsolateral prefrontal cortex } & 9 & $\mathrm{~L}$ & 6.759 & 0.010 & 5.658 & 0.019 \\
\hline & 9 & $\mathrm{R}$ & 39.628 & 0.000 & 36.657 & 0.000 \\
\hline & 46 & $L$ & 8.520 & 0.004 & 6.541 & 0.012 \\
\hline & 46 & $\mathrm{R}$ & 45.929 & 0.000 & 43.989 & 0.000 \\
\hline Inferior prefrontal gyrus & 47 & $\mathrm{R}$ & 24.570 & 0.000 & 25.746 & 0.000 \\
\hline Posterior entorhinal cortex & 28 & $\mathrm{~L}$ & - & - & 4.412 & 0.038 \\
\hline Temporopolar area & 38 & L & - & - & 5.617 & 0.019 \\
\hline
\end{tabular}

Notes: Brain regions are organized by functional implication into three sets of neural networks: (I) largely bilateral vision and somatosensory regions, (2) left-lateralized language regions, and (3) executive and memory regions. Brodmann's area, hemisphere, F-test, and significance test results from a one-way analysis of variance for each factor are provided.

Abbreviations: BA, Brodmann's area; hemi, hemisphere; L, left; R, right.

on the primary visual areas was engaged in the with prisms condition (yellow).

\section{Discussion}

VERPs were recorded from a patient with PTVS during two successive presentations of a pattern reversal task, the first when the patient did not wear corrective prisms and the second test when she wore corrective prisms. Using single-trial analysis of the VERP data, both conditions activated a P148 component that occurred between 120-256 milliseconds following each onset of the checkerboard pattern. A temporal principal components analysis identified differences in the VERPs that occurred between prism conditions at the end portion of the
P148 component that occurred between 168-256 milliseconds following the onset of the checkerboard. Scalp topographies indicated latency differences between conditions with peak negativity and positivity occurring $30-45$ milliseconds later in the no prisms condition. Thus, without corrective prisms, processing speed was slower, suggesting a less efficient visual system. Significantly different VERPs occurred during the two prism conditions as evidenced by their respective high classification accuracy scores obtained from the dynamic channel selection analysis. These differences occurred across all electrode sites and engaged different neural sources. During the no prisms condition, more neural sources were activated within the vision processing regions including the 
Table 3 Activation areas identified using GeoSource ${ }^{\circledR}$ software program (v2.0; Electrical Geodesics Inc, Eugene, OR) for with prisms condition

\begin{tabular}{|c|c|c|c|c|c|c|}
\hline \multirow[t]{2}{*}{ Region } & \multirow[t]{2}{*}{ BA } & \multirow[t]{2}{*}{ Hemi } & \multicolumn{2}{|c|}{ Factor 6} & \multicolumn{2}{|c|}{ Factor 9} \\
\hline & & & $F$ value & $P$ & F value & $\boldsymbol{P}$ \\
\hline \multicolumn{7}{|l|}{ Vision } \\
\hline Primary visual cortex (VI) & 17 & $\mathrm{R}$ & 5.298 & 0.023 & - & - \\
\hline Secondary visual cortex (V2) & 18 & $\mathrm{R}$ & 4.114 & 0.044 & - & - \\
\hline Associative visual cortex (V3) & 19 & $\mathrm{R}$ & 4.089 & 0.045 & - & - \\
\hline Hippocampus & $\mathrm{n} / \mathrm{a}$ & $\mathrm{R}$ & 6.291 & 0.013 & 5.083 & 0.026 \\
\hline Piriform cortex (AMY/HIPP) & 27 & $\mathrm{R}$ & 8.201 & 0.005 & 5.507 & 0.020 \\
\hline \multirow[t]{2}{*}{ Parahippocampal cortex } & 35 & $\mathrm{R}$ & 5.321 & 0.023 & - & - \\
\hline & 36 & $\mathrm{R}$ & 4.874 & 0.029 & 4.218 & 0.042 \\
\hline \multicolumn{7}{|l|}{ Memory and semantic } \\
\hline \multirow[t]{2}{*}{ Cingulate cortex } & 29 & $\mathrm{R}$ & 4.500 & 0.036 & - & - \\
\hline & 30 & $\mathrm{R}$ & 4.128 & 0.044 & - & - \\
\hline
\end{tabular}

Notes: Brain regions are organized by functional implication into two sets of neural networks: (I) right-lateralized vision regions, and (2) right-lateralized memory and semantic regions. Brodmann's area, hemisphere, F-test, and significance test results from a one-way analysis of variance for each factor are provided.

Abbreviations: AMY, amygdale; BA, Brodmann's area; hemi, hemisphere; HIPP, hippocampus; R, right.

primary somatosensory cortex and frontal eye fields. However, additional activations occurred in brain regions routinely implicated in language, memory, and executive functioning processing. Neurons fire approximately 10 milliseconds for every synapse (see Buonomano and Merzenich ${ }^{29}$ for evidence), so it is plausible that the recruitment of these additional brain regions required more time, thereby reducing processing speed as indicated by the delayed latency during the no prisms condition. In contrast, the with prisms condition engaged a more restricted neural network that included the primary visual regions, $\mathrm{V} 1, \mathrm{~V} 2$, and $\mathrm{V} 3$, in addition to brain regions believed to encode memories and semantic content, regions commonly implicated during typical vision processing. ${ }^{20}$

The behavioral changes associated with the use of prisms in this patient are vital to understanding the vast improvements in neural processing and general level of function. The sources

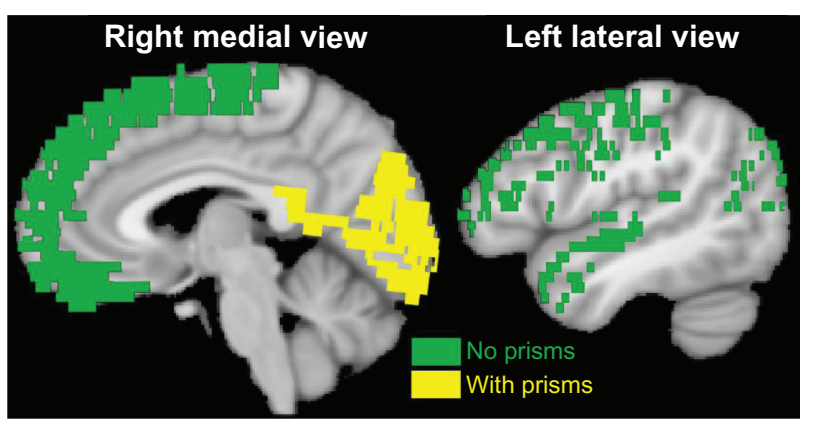

Figure 5 Source estimation difference maps for no prisms and with prisms conditions. Different patterns of activation were identified for both conditions using one-way analysis of variance between Brodmann's area sources. These patterns were superimposed on sagittal slices of the Montreal Neurological Institute template brain. Areas engaged during the no prisms condition are illustrated in green (notably, frontal, temporal, and parietal regions). Areas engaged during the with prisms condition are illustrated in yellow (notably, the right primary visual areas). estimated for the with prisms neural system implicated regions expected to be engaged during normal processing, notably the primary visual cortex. ${ }^{20}$ Considering that prisms are known to positively correct balance and posture in patients with head injuries, ${ }^{13}$ these results suggest that the prisms enabled the patient to activate the same neural mechanisms as a neurologically normal individual. Without prisms, the patient exhibited the symptoms of PTVS and VMSS, including poor balance and gross visual perceptual deficits. Problems with reading were likely also extensions of these deficits. A mismatch between the visual midline and proprioceptive input could result in an inability to focus on stationary objects such as print.

The source analyses indicated that the no prisms condition engaged brain areas normally thought to control language processes, including portions of Wernicke's and Broca's area. Consequently, in attempting to perform the visual reversal task, her brain engaged less optimal areas (auditory and language areas) to compensate for the reduced involvement of visual processing areas, thereby overloading the temporal and frontal lobes and interfering with their ability to support language functions. Stuttering, word recollection, and communication problems resulted. Such results provide new insights into how visual pathway mechanisms can impact higher order cognitive systems. To date, language and speech dysfunction related to PTVS and VMSS have not been addressed. Although prior work has implicated magnocellular deficits as contributing to language disorders such as dyslexia, ${ }^{30}$ other investigators suggest that unaffected populations have equal levels of deficits. ${ }^{31}$

These results support the view that neurooptometric rehabilitation using prisms significantly alters the 
engagement of neural mechanisms during a visual task. The process of recovery from TBI is not limited to compensatory mechanisms. ${ }^{32}$ Neurogenesis is possible late in development, ${ }^{33}$ and continued experiences drive cortical changes. ${ }^{29}$ Specific to visual processing, this study suggests that adjusting visual processing with corrective prisms impacts neural processing in measurable and meaningful ways. This intervention appears to better engage the visual system, facilitating the appropriate integration of information between the ambient and focal pathways and improving processing across proprioceptive, kinesthetic, vestibular, cognitive, and language domains.

Treatment for visual deficits is not a cure for widespread neurological dysfunction resulting from TBI. Symptom severity and other factors, including age at injury, bilateral damage, or substance abuse, may still lead to poor clinical prognosis. However, evidence here suggests that neurooptometric rehabilitation heavily influences the engagement of other sensory and cognitive systems. In addition, it is clear that electrophysiological recordings are useful in monitoring the progress of rehabilitation. ${ }^{16}$ This study also implicates VERP as a possible tool to specify prescription adjustments for corrective lenses and prisms, ensuring that optimal neural networks are engaged and supporting rehabilitation.

Despite these significant and exciting findings, there are several limitations to this study. First, without baseline information prior to the head injury, the VERP results can only address the subsequent neural processing that resulted from the injury. Second, while it is possible to quantify the effects of the prism, it is unknown whether any preexisting neurological conditions could have adversely affected the VERPs. Third, the condition order was not counterbalanced. It would be important to note if improvement in VERP organization and focused sources were due specifically to the order of presentation or to an increase in familiarity over the test period. Future work should counterbalance task order to determine whether equivalent patterns of brain activity occur.

Given the increase in identified cases of TBI, research involving the diagnosis, prognosis, and rehabilitation of brain injury is crucial. Research to date has primarily focused on specific symptoms of TBI, rather than elucidating the interactions between diverse neural mechanisms. As evident in this paper, symptoms specific to visual dysfunction can have a significant impact on the level of function across multiple domains. Once impairments are corrected, magnocellular ambient deficits appear to be reduced thereby eliminating inefficient compensatory mechanisms that may detract from other processes. Altogether, with the intervention imposed in this study, neural circuitry appears to become less atypical. Unfortunately, rehabilitation is not generally discussed until well after stabilization. More subtle symptoms such as PTVS or VMSS may not be identified until much later. VERP technology may provide an alternate and perhaps more effective guide for rehabilitation and treatment decisions, tracking ongoing progress, and predicting outcomes.

\section{Acknowledgments}

The authors thank their patient for her time and patience. They would also like to thank Nathan M Petro for his assistance in data collection and Madonna Rehabilitation Hospital for their cooperation. This work was supported in part by grants to DLM: NIH R43 HD062072, NIH 1R01EB007684, and DOE IEW R305K05186.

\section{Disclosure}

The authors report no conflicts of interest in this work.

\section{References}

1. Rutland-Brown W, Langlois JA, Thomas KE, Xi YL. Incidence of traumatic brain injury in the United States, 2003. J Head Trauma Rehabil. 2006;21(6):544-548.

2. Oppenheimer DR. Microscopic lesions in the brain following head injury. J Neurol Neurosurg Psychiatry. 1968;31(4):299-306.

3. Levin HS, Amparo E, Eisenberg HM, et al. Magnetic resonance imaging and computerized tomography in relation to the neurobehavioral sequelae of mild and moderate head injuries. $J$ Neurosurg. 1987;66(5):706-713.

4. Singh M, Jeong J, Hwang D, Sungkarat W, Gruen P. Novel diffusion tensor imaging methodology to detect and quantify injured regions and affected brain pathways in traumatic brain injury. Magn Reson Imaging. 2010;28(1):22-40.

5. Schlageter K, Gray B, Hall K, Shaw R, Sammet R. Incidence and treatment of visual dysfunction in traumatic brain injury. Brain Inj. 1993;7(5):439-448.

6. Houwen S, Hartman E, Visscher C. Physical activity and motor skills in children with and without visual impairments. Med Sci Sports Exerc. 2009;41(1):103-109.

7. Legault I, Gagne JP, Rhoualem W, Anderson-Gosselin P. The effects of blurred vision on auditory-visual speech perception in younger and older adults. Int J Audiol. 2010;49(12):904-911.

8. Hellerstein LF, Freed S, Maples WC. Vision profile of patients with mild brain injury. J Am Optom Assoc. 1995;66(10):634-639.

9. Padula WV, Argyris S, Ray J. Visual evoked potentials (VEP) evaluating treatment for post-trauma vision syndrome (PTVS) in patients with traumatic brain injuries (TBI). Brain Inj. 1994;8(2):125-133.

10. Padula WV, Shapiro JB, Argyris S. The effect of post trauma vision syndrome (PTVS) and visual midline shift syndrome (VMSS) on function following a neurological event. In: Stuen C, Arditi A, Horowitz A, Lang MA, Rosenthal B, Seidman KR, editors. Vision Rehabilitation: Assessment, Intervention, and Outcomes. Lisse: Swets and Zeitlinger Publishers; 2000:114-116.

11. Malamut $\mathrm{D}$. Vestibular therapy and ocular dysfunction in traumatic brain injury: a case study. In: Suchoff IB, Kapoor N, Ciuffreda KJ, editors. Visual and Vestibular Consequences of Acquired Brain Injuries. Santa Ana, CA: Optometric Extension Program; 2001:201-220. 
12. Rosen SA, Cohen AH, Trebing S. The integration of visual and vestibular systems in balance disorders - a clinical perspective. In: Suchoff IB, Kapoor N, Ciuffreda KJ, editors. Visual and Vestibular Consequences of Acquired Brain Injuries. Santa Ana, CA: Optometric Extension Program; 2001:174-200.

13. Padula WV, Nelson CA, Benabib R, Yilmaz T, Krevisky S. Modifying postural adaptation following a CVA through prismatic shift of visuospatial egocenter. Brain Inj. 2009;23(6):566-576.

14. Dockree PM, Robertson IH. Electrophysiological markers of cognitive deficits in traumatic brain injury: a review. Int J Psychophysiol. 2011 82(1):53-60.

15. Duncan CC, Summers AC, Perla EJ, Coburn KL, Mirsky AF. Evaluation of traumatic brain injury: brain potentials in diagnosis, function, and prognosis. Int J Psychophysiol. 2011;82(1):24-40.

16. Folmer RL, Billings CJ, Diedesch-Rouse AC, Gallun FJ, Lew HL. Electrophysiological assessments of cognition and sensory processing in TBI: applications for diagnosis, prognosis and rehabilitation. Int $J$ Psychophysiol. 2011;82(1):4-15.

17. Sarno S, Erasmus LP, Lippert G, Frey M, Lipp B, Schlaegel W. Electrophysiological correlates of visual impairments after traumatic brain injury. Vision Res. 2000;40(21):3029-3038.

18. Keane S, Turner C, Sherrington C, Beard JR. Use of fresnel prism glasses to treat stroke patients with hemispatial neglect. Arch Phys Med Rehabil. 2006;87(12):1668-1672.

19. Rossi PW, Kheyfets S, Reding MJ. Fresnel prisms improve visual perception in stroke patients with homonymous hemianopia or unilateral visual neglect. Neurology. 1990;40(10):1597-1599.

20. Di Russo F, Pitzalis S, Spitoni G, et al. Identification of the neural sources of the pattern-reversal VEP. Neuroimage. 2005;24(3):874-886.

21. Stewart WF, Lipton RB, Kolodner K, Liberman J, Sawyer J. Reliability of the migraine disability assessment score in a population-based sample of headache sufferers. Cephalalgia. 1999;19(2):107-114.

22. Oldfield RC. The assessment and analysis of handedness: the Edinburgh inventory. Neuropsychologia. 1971;9(1):97-1 13.
23. Colarusso R, Hammill D. Motor-Free Visual Perception Test-Revised. Los Angeles, CA: Western Psychological Service; 1995.

24. Cate Y, Richards L. Relationship between performance on tests of basic visual functions and visual-perceptual processing in persons after brain injury. Am J Occup Ther. 2000;54(3):326-334.

25. Rockstroh B, Elbert T, Lutzenberger W, Birbaumer N. The effects of slow cortical potentials on response speed. Psychophysiology. 1982;19(2):211-217.

26. Kaiser HF, Rice J. Little Jiffy, Mark IV. Educ Psychol Meas. 1974;34 111-117.

27. Kota S, Gupta L, Molfese DL, Vaidyanathan R. A dynamic channel selection strategy for dense-array ERP classification. IEEE Trans Biomed Eng. 2009;56(4):1040-1051.

28. Molfese DL, Molfese VJ, Beswick J, et al. Dynamic links between emerging cognitive skills and brain processes. Dev Neuropsychol. 2008;33(6):682-706.

29. Buonomano DV, Merzenich MM. Cortical plasticity: from synapses to maps. Annu Rev Neurosci. 1998;21:149-186.

30. Johnston A, Bruno A, Watanabe J, et al. Visually-based temporal distortion in dyslexia. Vision Res. 2008;48(17):1852-1858.

31. Skoyles J, Skottun BC. On the prevalence of magnocellular deficits in the visual system of non-dyslexic individuals. Brain Lang. 2004;88(1):79-82.

32. Kerkhoff G. Neurovisual rehabilitation: recent developments and future directions. Am J Ophthalmol. 2000;130(5):687-688.

33. McMillan T, Jacobson RR. Traumatic brain injury and post-traumatic stress disorder. Br J Psychiatry. 1999;174:274-275.

34. Curran T. The electrophysiology of incidental and intentional retrieval: ERP old/new effects in lexical decision and recognition memory. Neuropsychologia. 1999;37(7):771-785.

35. Ferree TC, Eriksen KJ, Tucker DM. Regional head tissue conductivity estimation for improved EEG analysis. IEEE Trans Biomed Eng. 2000;47(12):1584-1592. 


\section{Supplementary data}

\section{Stimuli design and procedure}

Stimuli consisted of alternating pattern reversals of an $8 \times 8$ grid of $3.2 \mathrm{~cm}^{2}$ black-and-white checkerboards at a visual angle of 17.1 degrees horizontal $\times 17.1$ degrees vertical on a gray background. A total of 100 trials were presented on a gray background for 1000 milliseconds each. Stimuli were displayed using E-Prime ${ }^{\circledR}$ software (v2.0; Psychological Software Tools Inc, Pittsburgh, PA) on a separate computer that integrated with Net Station 4.4.2 software (Electric Geodesics Inc, Eugene, OR) to mark events on the ongoing electroencephalographic data collection stream. The subject was seated $1 \mathrm{~m}$ away from a Dell 20.5" liquid crystal display monitor (Dell Inc, Austin, TX) that displayed the checkerboard patterns.

\section{Event-related potential data preprocessing procedure}

Electrode impedances recorded before and after the task were below $60 \mathrm{k} \mathrm{ohm}$ to maximize signal-to-noise ratio, producing high-quality signals for subsequent analyses. The ongoing electroencephalographic signals were digitized at 4 milliseconds intervals for each of the 256 electrode sites. High-pass filters were set to $0.1 \mathrm{~Hz}$ and low-pass filters to $30 \mathrm{~Hz}$ with a gain of $10 \mathrm{k} \mathrm{ohm}$. Single-trial epochs were segmented from continuous electroencephalography to create epochs from the exact onset of the checkerboard pattern (0 milliseconds) to 800 milliseconds following onset. Voltage shifts greater than $100 \mu \mathrm{V}$ during the epoch (for instance, due to eye movements or blinks) were classified as artifacts. For trials and electrode channels on which artifacts were detected, the event-related potential signal was deleted and replaced with an average signal interpolated from immediately adjacent electrode locations following standard procedures. Remaining epochs were then baseline corrected, referenced again to the average of all electrodes, and then clustered into 18 scalp regions (orbital, inferior frontal, prefrontal, inferior temporal, temporal, temporoparietal, parietal, inferior occipital, and occipital for both the left and right hemisphere). The purpose of this approach was to reduce the number of variables in order to increase statistical power for the planned single trail analyses ${ }^{34}$ Epochs were paired across runs (with and without prisms) to ensure that the same trial numbers were considered. For example, trial 88 contained an artifact for the no prisms condition, so trial 88 was removed from analyses for both conditions.

\section{Event-related potential brain source analysis methods}

A finite difference model (FDM) was applied using a forward modeling approach to accurately compute the electrode locations in relation to brain tissues. Finite difference model estimates were constrained by the Montreal Neurological Institute average adult magnetic resonance imaging database. Tissue volumes were parceled using 7-mm voxels, each serving as a dipole source location with three orthogonal orientations (in $\mathrm{x}, \mathrm{y}$, and $\mathrm{z}$ orientations). The finite difference model applied estimations across a total of 2447 source dipole triplets. Conductivity values used in the finite difference model included $0.25 \mathrm{~S} / \mathrm{m}$ for brain, $1.8 \mathrm{~S} / \mathrm{m}$ for cerebral spinal fluid, $0.018 \mathrm{~S} / \mathrm{m}$ for skull, and $0.44 \mathrm{~S} / \mathrm{m}$ for scalp..$^{35}$ Weighting was placed equally across locations with regularization carried out via Tikhonov $\left(1 \times 10^{-2}\right)$ using standardized low resolution brain electromagnetic tomography as a constraint.
Eye and Brain

\section{Publish your work in this journal}

Eye and Brain is an international, peer-reviewed, open access journal focusing on clinical and experimental research in the field of neuro-ophthalmology. All aspects of patient care are addressed within the journal as well as basic research. Papers covering original research, basic science, clinical and epidemiological studies, reviews and evaluations,

\section{Dovepress}

guidelines, expert opinion and commentary, case reports and extended reports are welcome. The manuscript management system is completely online and includes a very quick and fair peer-review system, which is all easy to use. Visit http://www.dovepress.com/testimonials.php to read real quotes from published authors. 